\title{
Generalized order of entire monogenic functions of slow growth
}

\author{
Susheel Kumar*, Kirandeep Bala \\ Department of Mathematics, Central University of Himachal Pradesh, Dharamshala-176215, INDIA. \\ Dedicated to George A Anastassiou on the occasion of his sixtieth birthday \\ Communicated by Professor Gh. Sadeghi
}

\begin{abstract}
In the present paper we study the generalized growth of entire monogenic functions having slow growth. The characterizations of generalized order of entire monogenic functions have been obtained in terms of their Taylor's series coefficients.
\end{abstract}

Keywords: Clifford algebra, Clifford analysis, Generalized Cauchy-Riemann system, Entire monogenic function, Generalized order.

2010 MSC: Primary 30G35; Secondary 30D15.

\section{Introduction}

Clifford analysis offers possibility of generalizing complex function theory to higher dimensions. It considers Clifford algebra valued functions that are defined in open subsets of $\mathbb{R}^{n}$ for arbitrary finite $n \in \mathbb{N}$ and that are solutions of higher dimensional Cauchy-Riemann systems. These are often called Clifford holomorphic or monogenic functions.

In order to make calculations more concise we use the following notations, where $\mathbf{m}=\left(m_{1}, \ldots, m_{n}\right) \in \mathbb{N}_{0}^{n}$ is the $n$-dimensional multi-index and $\mathbf{x} \in \mathbb{R}^{n}$ :

$$
\mathbf{x}^{\mathbf{m}}=x_{1}^{m_{1}} \ldots x_{n}^{m_{n}}, \quad \mathbf{m} !=m_{1} ! \ldots m_{n} !, \quad|\mathbf{m}|=m_{1}+\ldots+m_{n} .
$$

Following Constales, Almeida and Krausshar (see [1] and [2]), we give some definitions and associated properties.

\footnotetext{
* Corresponding author

Email addresses: sus83dma@gmail.com (Susheel Kumar), kirandeepbala86@gmail.com (Kirandeep Bala)
} 
By $\left\{e_{1}, e_{2}, \ldots, e_{n}\right\}$ we denote the canonical basis of the Euclidean vector space $\mathbb{R}^{n}$. The associated real Clifford algebra $C l_{0 n}$ is the free algebra generated by $\mathbb{R}^{n}$ modulo $\mathbf{x}^{2}=-\|\mathbf{x}\|^{2} e_{0}$, where $e_{0}$ is the neutral element with respect to multiplication of the Clifford algebra $C l_{0 n}$. In the Clifford algebra $C l_{0 n}$ following multiplication rule holds:

$$
e_{i} e_{j}+e_{j} e_{i}=-2 \delta_{i j} e_{0}, \quad i, j=1,2, \ldots, n,
$$

where $\delta_{i j}$ is Kronecker symbol. A basis for Clifford algebra $C l_{0 n}$ is given by the set $\left\{e_{A}: A \subseteq\{1,2, \ldots, n\}\right\}$ with $e_{A}=e_{l_{1}} e_{l_{2}} \ldots e_{l_{r}}$, where $1 \leq l_{1}<l_{2}<\ldots<l_{r} \leq n, e_{\phi}=e_{0}=1$. Each $a \in C l_{0 n}$ can be written in the form $a=\sum_{A} a_{A} e_{A}$ with $a_{A} \in \mathbb{R}$. The conjugation in Clifford algebra $C l_{0 n}$ is defined by $\bar{a}=\sum_{A} a_{A} \bar{e}_{A}$, where

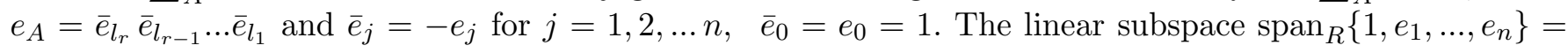
$\mathbb{R} \oplus \mathbb{R}^{n} \subset C l_{0 n}$ is the so called space of paravectors $z=x_{0}+x_{1} e_{1}+x_{2} e_{2}+\ldots+x_{n} e_{n}$ which we simply identify with $\mathbb{R}^{n+1}$. Here $x_{0}=\operatorname{Sc}(z)$ is scalar part and $\mathbf{x}=x_{1} e_{1}+x_{2} e_{2}+\ldots+x_{n} e_{n}=\operatorname{Vec}(z)$ is vector part of paravector $z$. The Clifford norm of an arbitrary $a=\sum_{A} a_{A} e_{A}$ is given by

$$
\|a\|=\left(\sum_{A}\left|a_{A}\right|^{2}\right)^{1 / 2} .
$$

Each paravector $z \in \mathbb{R}^{n+1} \backslash\{0\}$ has an inverse element in $\mathbb{R}^{n+1}$ which can be represented in the form $z^{-1}=\bar{z} /\|z\|^{2}$.

The generalized Cauchy-Riemann operator in $\mathbb{R}^{n+1}$ is given by

$$
D \equiv \frac{\partial}{\partial x_{0}}+\sum_{i=1}^{n} e_{i} \frac{\partial}{\partial x_{i}} .
$$

If $U \subseteq \mathbb{R}^{n+1}$ is an open set, then a function $g: U \rightarrow C l_{0 n}$ is called left (right) monogenic at a point $z \in U$ if $D g(z)=0(g D(z)=0)$. The functions which are left (right) monogenic in the whole space are called left (right) entire monogenic functions.

Let $A_{n+1}$ be the $n$-dimensional surface area of $n+1$-dimensional unit ball and $q_{0}(z)=\bar{z} /\|z\|^{n+1}$ be Cauchy kernel function. Then every function $g$ which is monogenic in a neighborhood of closure $\bar{G}$ of domain $G$ satisfies the following equation (see [2], p. 766)

$$
g(z)=\frac{1}{A_{n+1}} \int_{\partial G} q_{0}(z-\zeta) d \tau(\zeta) g(\zeta), \text { for all } z \in G,
$$

where

$$
d \tau(\zeta)=\sum_{j=0}^{n}(-1)^{j} e_{j} \widehat{d \zeta}_{j}
$$

with

$$
\widehat{d \zeta}_{j}=d \zeta_{0} \wedge \ldots \wedge d \zeta_{j-1} \wedge d \zeta_{j+1} \wedge \ldots \wedge d \zeta_{n}
$$

is the oriented outer normal surface measure. Following [1] and [2], we define the Fueter polynomials $V_{\mathbf{m}}(z)$ as

$$
V_{\mathbf{m}}(z)=\frac{\mathbf{m} !}{|\mathbf{m}| !} \sum_{\pi \in \operatorname{perm}(\mathbf{m})} z_{\pi\left(m_{1}\right)} \ldots z_{\pi\left(m_{n}\right)},
$$

where $\operatorname{perm}(\mathbf{m})$ is the set of all permutations of the sequence $\left(m_{1}, m_{2} \ldots, m_{n}\right)$ and $z_{i}=x_{i}-x_{0} e_{i}$ for $i=1, \ldots, n$ and $V_{\mathbf{0}}(z)=1$. If $g$ is a left monogenic function in a ball $\|z\|<R$, then for all $\|z\|<r$ with $0<r<R$, the Taylor series expansion of $g(z)$ is given by (see [1] and [2]) 


$$
g(z)=\sum_{|\mathbf{m}|=0}^{\infty} V_{\mathbf{m}}(z) a_{\mathbf{m}} .
$$

In (1.1), $\left\{a_{\mathbf{m}}\right\}$ are Clifford numbers which are defined by

$$
a_{\mathbf{m}}=\frac{1}{\mathbf{m} ! A_{n+1}} \int_{\|\zeta\|<r} q_{\mathbf{m}}(\zeta) d \tau(\zeta) g(\zeta)
$$

and satisfy the inequality

$$
\left\|a_{\mathbf{m}}\right\| \leq c(n, \mathbf{m}) \frac{M(r, g)}{r^{|\mathbf{m}|}} .
$$

Here $M(r, g)=\max _{\|z\|=r}\{\|g(z)\|\}$ denotes the maximum modulus of the function $g$ in the closed ball of radius $r$ and

$$
q_{\mathbf{m}}(z)=\frac{\partial^{m_{0}+m_{1}+\ldots+m_{n}}}{\partial x_{0}^{m_{0}} \partial x_{1}^{m_{1}} \ldots \partial x_{n}^{m_{n}}} q_{\mathbf{0}}(z), \quad c(n, \mathbf{m})=\frac{n(n+1) \ldots(n+|\mathbf{m}|-1)}{\mathbf{m} !} .
$$

The concept of generalized order and generalized type for entire transcendental functions was given by Seremeta [4], Kapoor and Nautiyal [3]. Hence, let $L^{0}$ denote the class of functions $h(x)$ satisfying the following conditions:

(i) $h(x)$ is defined on $[a, \infty)$ and is positive, strictly increasing, differentiable and tends to $\infty$ as $x \rightarrow \infty$,

(ii) $\lim _{x \rightarrow \infty} \frac{h[\{1+1 / \psi(x)\} x]}{h(x)}=1$, for every function $\psi(x)$ such that $\psi(x) \rightarrow \infty$ as $x \rightarrow \infty$. The functions of the form $f(x)=a x+b, 0<a<\infty, 0<b<\infty$ are in class $L^{0}$.

Let $\Lambda$ denote the class of functions $h$ satisfying conditions (i) and

(iii) $\lim _{x \rightarrow \infty} \frac{h(c x)}{h(x)}=1$, for every $c>0$, that is $h(x)$ is slowly increasing. The functions of the form $f(x)=$ $\log (a x), 0<a<\infty$, are in class $\Lambda$.

Let $\Omega$ be the class of functions $h(x)$ satisfying conditions (i) and

(iv) there exist a function $\delta(x) \in \Lambda$ and constants $x_{0}, K_{1}$ and $K_{2}$ such that

$$
0<K_{1} \leq \frac{d\{h(x)\}}{d\{\delta(\log x)\}} \leq K_{2}<\infty
$$

for all $x>x_{0}$. The functions of the form $f(x)=\delta(\log x), \delta \in \Lambda$ are in class $\Omega$. (see [3] )

Let $\bar{\Omega}$ be the class of functions $h(x)$ satisfying (i) and

(v) $\lim _{x \rightarrow \infty} \frac{d\{h(x)\}}{d(\log x)}=K, \quad 0<K<\infty$. The functions of the form $f(x)=\log x+a(\log \log x)^{b}, 0<a<\infty, 0<$ $b<\infty$ are in class $\bar{\Omega}$. (see [3])

Kapoor and Nautiyal [3] showed that classes $\Omega$ and $\bar{\Omega}$ are contained in $\Lambda$ and $\Omega \bigcap \bar{\Omega}=\phi$.

For an entire monogenic function $g(z)$ and functions $\alpha(x)$ either belongs to $\Omega$ or to $\bar{\Omega}$, we define the generalized order $\rho(\alpha, g)$ of $g(z)$ as

$$
\rho(\alpha, g)=\lim _{r \rightarrow \infty} \sup \frac{\alpha[\log M(r, g)]}{\alpha(\log r)} .
$$




\section{Main results}

We now prove

Theorem 2.1. Let $g: \mathbb{R}^{n+1} \rightarrow C l_{0 n}$ be an entire monogenic function whose Taylor's series representation is given by $g(z)=\sum_{|\boldsymbol{m}|=0}^{\infty} V_{\boldsymbol{m}}(z) a_{\boldsymbol{m}}$. If $\alpha(x)$ either belongs to $\Omega$ or to $\bar{\Omega}$, then the generalized order $\rho(\alpha, g)(1<$ $\rho(\alpha, g)<\infty)$ of $g(z)$ is given as

$$
\rho(\alpha, g)-1=\lim _{|\boldsymbol{m}| \rightarrow \infty} \sup \frac{\alpha(|\boldsymbol{m}|)}{\alpha\left\{\log \left\|a_{\boldsymbol{m}} / c(n, \boldsymbol{m})\right\|^{-1 /|\boldsymbol{m}|}\right\}} .
$$

Proof. Write

$$
\theta=\lim _{|\mathbf{m}| \rightarrow \infty} \sup \frac{\alpha(|\mathbf{m}|)}{\alpha\left\{\log || a_{\mathbf{m}} / c(n, \mathbf{m}) \|^{-1 /|\mathbf{m}|}\right\}} .
$$

Now first we prove that $\rho-1 \geq \theta$. The coefficients of a monogenic Taylor's series satisfy Cauchy's inequality, that is

$$
\left\|a_{\mathbf{m}} / c(n, \mathbf{m})\right\| \leq r^{-|\mathbf{m}|} M(r, g) .
$$

Also from 1.2 , for $\varepsilon>0$ and all $r>r_{0}(\varepsilon)$, we have

$$
M(r, g) \leq \exp \left[\alpha^{-1}\{\bar{\rho} \alpha(\log r)\}\right],
$$

where $\bar{\rho}=\rho+\varepsilon$ provided $r$ is sufficiently large. So from 2.2 , we get

$$
\left\|a_{\mathbf{m}} / c(n, \mathbf{m})\right\| \leq r^{-|\mathbf{m}|} \exp \left[\alpha^{-1}\{\bar{\rho} \alpha(\log r)\}\right]
$$

or

$$
\left\|a_{\mathbf{m}} / c(n, \mathbf{m})\right\| \leq \exp \left[-|\mathbf{m}| \log r+\alpha^{-1}\{\bar{\rho} \alpha(\log r)\}\right] .
$$

Since $\alpha(x)$ is an increasing function of $x$, we define $r=r(|\mathbf{m}|)$ as the unique root of the equation

$$
\alpha\left[\frac{|\mathbf{m}| \log r}{\bar{\rho}}\right]=\bar{\rho} \alpha(\log r) .
$$

For large values of $|\mathbf{m}|$, we have

$$
\begin{aligned}
& \alpha(c|\mathbf{m}|) \simeq \alpha(|\mathbf{m}|) \\
& \Rightarrow \alpha(c|\mathbf{m}|) \simeq \alpha(|\mathbf{m}|)\{1+o(1)\} \\
& \Rightarrow \alpha(c|\mathbf{m}|) \simeq \alpha(|\mathbf{m}|)\left\{1+\frac{\alpha(c)}{\alpha(|\mathbf{m}|)}\right\} \\
& \Rightarrow \alpha(c|\mathbf{m}|) \simeq \alpha(|\mathbf{m}|)+\alpha(c) .
\end{aligned}
$$

Thus for large values of $|\mathbf{m}|$ from equation (2.4), we have

$$
\bar{\rho} \alpha(\log r) \simeq \alpha(|\mathbf{m}|)+\alpha(\log r)-\alpha(\bar{\rho})
$$

or

or

$$
\alpha(\log r) \simeq \frac{\alpha(|\mathbf{m}|)}{(\bar{\rho}-1)}\left\{1-\frac{\alpha(\bar{\rho})}{\alpha(|\mathbf{m}|)}\right\} .
$$

$$
\alpha(\log r) \simeq \frac{\alpha(|\mathbf{m}|)}{(\bar{\rho}-1)}\{1+o(1)\}
$$


or

$$
\log r \simeq \alpha^{-1}\left\{\frac{1}{\bar{\rho}-1} \alpha(|\mathbf{m}|)\right\}=F\left(|\mathbf{m}|, \frac{1}{\bar{\rho}-1}\right) .
$$

Using (2.4) and 2.5) in 2.3), we get

$$
\left\|a_{\mathbf{m}} / c(n, \mathbf{m})\right\| \leq \exp [-|\mathbf{m}| F+(|\mathbf{m}| / \bar{\rho}) F]
$$

or

or

$$
\frac{\bar{\rho}}{\bar{\rho}-1} \log \left\{\| a_{\mathbf{m}} / c(n, \mathbf{m})||\right\}^{-1 /|\mathbf{m}|} \geq \alpha^{-1}\left\{\frac{1}{\bar{\rho}-1} \alpha(|\mathbf{m}|)\right\}
$$

$$
\frac{\alpha(|\mathbf{m}|)}{\alpha\left[\frac{\bar{\rho}}{\bar{\rho}-1} \log \left\{\left\|a_{\mathbf{m}} / c(n, \mathbf{m})\right\|\right\}^{-1 /|\mathbf{m}|}\right]} \leq \bar{\rho}-1
$$

or

$$
\begin{aligned}
\frac{\alpha(|\mathbf{m}|)}{\alpha\left[\log \left\{\left\|a_{\mathbf{m}} / c(n, \mathbf{m})\right\|\right\}^{-1 /|\mathbf{m}|}\right]} \leq & (\bar{\rho}-1) \times \\
& \times \frac{\alpha\left[\frac{\bar{\rho}}{\bar{\rho}-1} \log \left\{\left\|a_{\mathbf{m}} / c(n, \mathbf{m})\right\|\right\}^{-1 /|\mathbf{m}|]}\right.}{\alpha\left[\log \left\{\left\|a_{\mathbf{m}} / c(n, \mathbf{m})\right\|\right\}^{-1 /|\mathbf{m}|}\right]} .
\end{aligned}
$$

Since $\alpha(c x) \simeq \alpha(x)$ as $x \rightarrow \infty$, proceeding to limits as $|\mathbf{m}| \rightarrow \infty$ we get

$$
\theta \leq \bar{\rho}-1
$$

Since $\varepsilon>0$ is arbitrarily small, we finally get

$$
\theta \leq \rho-1 .
$$

Now we will prove that $\theta \geq \rho-1$. If $\theta=\infty$, then there is nothing to prove. So let us assume that $0 \leq \theta<\infty$. Therefore, for all $\varepsilon>0$ there exist $n_{0} \in N$ such that for all multi-indices $\mathrm{m}$ with $|\mathbf{m}|>n_{0}$, we have

or

$$
0 \leq \frac{\alpha(|\mathbf{m}|)}{\alpha\left[\log \left\{\left\|a_{\mathbf{m}} / c(n, \mathbf{m})\right\|\right\}^{-1 /|\mathbf{m}|}\right]}<\theta+\varepsilon=\bar{\theta}
$$

$$
\left\|a_{\mathbf{m}} / c(n, \mathbf{m})\right\| \leq \exp \left[-|\mathbf{m}| \alpha^{-1}\{\alpha(|\mathbf{m}|) / \bar{\theta}\}\right] .
$$

Now from the property of maximum modulus, we have

$$
M(r, g) \leq \sum_{|\mathbf{m}|=0}^{\infty} \| a_{\mathbf{m}}|| r^{|\mathbf{m}|}
$$

or

$$
\begin{aligned}
M(r, g) & \leq \sum_{|\mathbf{m}|=0}^{n_{0}}|| a_{\mathbf{m}}|| r^{|\mathbf{m}|}+ \\
& +\sum_{|\mathbf{m}|=n_{0}+1}^{\infty} c(n, \mathbf{m}) r^{|\mathbf{m}|} \exp \left[-|\mathbf{m}| \alpha^{-1}\{\alpha(|\mathbf{m}|) / \bar{\theta}\}\right] .
\end{aligned}
$$

Now for $r=\max \left\{1, \exp \left(\alpha^{-1}\left(\frac{\alpha\left(n_{0}+1\right)}{\bar{\theta}}\right) /(n+1)\right)\right\}$, we have

$$
\begin{aligned}
& M(r, g) \leq A_{1} r^{n_{0}}+ \\
& \quad+\sum_{|\mathbf{m}|=n_{0}+1}^{\infty} c(n, \mathbf{m}) r^{|\mathbf{m}|} \exp \left[-|\mathbf{m}| \alpha^{-1}\{\alpha(|\mathbf{m}|) / \bar{\theta}\}\right],
\end{aligned}
$$


where $A_{1}$ is a positive real constant. We take

$$
N(r)=\left[\alpha^{-1}\{\bar{\theta} \alpha[\log \{(n+1) r\}]\}\right],
$$

where $[x]$ denotes the integer part of $x \geq 0$. Since $\alpha(x)$ either belongs to $\Omega$ or to $\bar{\Omega}$, the integer $N(r)$ is well defined. Now if $r$ is sufficiently large, then from (2.7) we have

$$
\begin{aligned}
& M(r, g) \leq A_{1} r^{n_{0}}+r^{N(r)} \times \\
& \times \sum_{n_{0}+1 \leq|\mathbf{m}| \leq N(r)} c(n, \mathbf{m}) \exp \left[-|\mathbf{m}| \alpha^{-1}\{\alpha(|\mathbf{m}|) / \bar{\theta}\}\right] \\
&+\sum_{|\mathbf{m}|>N(r)} c(n, \mathbf{m}) r^{|\mathbf{m}|} \exp \left[-|\mathbf{m}| \alpha^{-1}\{\alpha(|\mathbf{m}|) / \bar{\theta}\}\right]
\end{aligned}
$$

or

$$
\begin{aligned}
& M(r, g) \leq A_{1} r^{n_{0}}+r^{N(r)} \times \\
& \times \sum_{|\mathbf{m}|=1}^{\infty} c(n, \mathbf{m}) \exp \left[-|\mathbf{m}| \alpha^{-1}\{\alpha(|\mathbf{m}|) / \bar{\theta}\}\right] \\
&+\sum_{|\mathbf{m}|>N(r)} c(n, \mathbf{m}) r^{|\mathbf{m}|} \exp \left[-|\mathbf{m}| \alpha^{-1}\{\alpha(|\mathbf{m}|) / \bar{\theta}\}\right] .
\end{aligned}
$$

Now the first series in 2.8 can be rewritten as

$$
\sum_{p=1}^{\infty}\left(\sum_{|\mathbf{m}|=p} c(n, \mathbf{m})\right) \exp \left[-p \alpha^{-1}\{\alpha(p) / \bar{\theta}\}\right] .
$$

Now from ([2], Lemma 1), we have

$$
\lim _{p \rightarrow \infty} \sup \left(\sum_{|\mathbf{m}|=p} c(n, \mathbf{m})\right)^{1 / p}=n .
$$

Hence we have

$$
\begin{aligned}
& \lim _{p \rightarrow \infty} \sup \left[\left(\sum_{|\mathbf{m}|=p} c(n, \mathbf{m})\right) \exp \left[-p \alpha^{-1}\{\alpha(p) / \bar{\theta}\}\right]\right]^{1 / p} \\
& =n \lim _{p \rightarrow \infty} \sup \exp \left[-\alpha^{-1}\{\alpha(p) / \bar{\theta}\}\right]=0 .
\end{aligned}
$$

Hence the series (2.9) converges to a positive real constant $A_{2}$. So from (2.8), we get

$$
\begin{aligned}
& M(r, g) \leq A_{1} r^{n_{0}}+A_{2} r^{N(r)}+ \\
& \quad+\sum_{|\mathbf{m}|>N(r)} c(n, \mathbf{m}) r^{|\mathbf{m}|} \exp \left[-|\mathbf{m}| \alpha^{-1}\{\alpha(|\mathbf{m}|) / \bar{\theta}\}\right]
\end{aligned}
$$

or

$$
\begin{aligned}
& M(r, g) \leq A_{1} r^{n_{0}}+A_{2} r^{N(r)}+ \\
& \quad+\sum_{|\mathbf{m}|>N(r)} c(n, \mathbf{m}) r^{|\mathbf{m}|} \exp [-|\mathbf{m}| \log \{(n+1) r\}]
\end{aligned}
$$

or

$$
M(r, g) \leq A_{1} r^{n_{0}}+A_{2} r^{N(r)}+\sum_{|\mathbf{m}|>N(r)} c(n, \mathbf{m})\left(\frac{1}{n+1}\right)^{|\mathbf{m}|}
$$

or

$$
M(r, g) \leq A_{1} r^{n_{0}}+A_{2} r^{N(r)}+\sum_{|\mathbf{m}|=1}^{\infty} c(n, \mathbf{m})\left(\frac{1}{n+1}\right)^{|\mathbf{m}|} .
$$

The series in 2.10$)$ can we rewritten as 


$$
\sum_{p=1}^{\infty}\left(\sum_{|\mathbf{m}|=p} c(n, \mathbf{m})\right)\left(\frac{1}{n+1}\right)^{p}
$$

So we have

$$
\lim _{p \rightarrow \infty} \sup \left[\left(\sum_{|\mathbf{m}|=p} c(n, \mathbf{m})\right)\left\{\frac{1}{n+1}\right\}^{p}\right]^{1 / p}=\frac{n}{n+1}<1 .
$$

Hence the series (2.11) converges to a positive real constant $A_{3}$. Therefore from 2.10), we get

$$
M(r, g) \leq A_{1} r^{n_{0}}+A_{2} r^{N(r)}+A_{3} .
$$

Since $N(r) \rightarrow \infty$ as $r \rightarrow \infty$ so we can write above inequality as

$$
\log M(r, g) \leq[1+o(1)] N(r) \log r
$$

or

$$
\begin{aligned}
\log M(r, g) \leq & {[1+o(1)]\left[\alpha^{-1}\{\bar{\theta} \alpha[\log \{(n+1) r\}]\}\right] \log r } \\
\leq & {[1+o(1)]\left[\alpha^{-1}\{\bar{\theta} \alpha[\log \{(n+1) r\}]\}\right] \times } \\
& \times\left[\alpha^{-1}\{\alpha[\log \{(n+1) r\}]\}\right] \\
\leq & {[1+o(1)]\left[\alpha^{-1}\{(\bar{\theta}+1) \alpha[\log \{(n+1) r\}]\}\right] }
\end{aligned}
$$

or

$$
\alpha[\log M(r, g)] \leq(\bar{\theta}+1) \alpha[\log \{(n+1) r\}]
$$

or

$$
\frac{\alpha[\log M(r, g)]}{\alpha(\log r)} \leq(\bar{\theta}+1) \quad \frac{\alpha[\{1+o(1)\} \log r]}{\alpha(\log r)} .
$$

Proceeding to limits as $r \rightarrow \infty$ and using properties of $\alpha(x)$, we get

$$
\rho \leq \bar{\theta}+1
$$

Since $\varepsilon>0$ is arbitrarily small, we finally get

$$
\rho-1 \leq \theta .
$$

Combining (2.6) and 2.12), we get 2.1). Hence Theorem 2.1 is proved.

\section{Acknowledgements:}

The authors are very thankful to the referee for his valuable comments and observations which helped in improving the paper. 


\section{References}

[1] D. Constales, R. De Almeida and R. S. Krausshar, On the growth type of entire monogenic functions, Arch. Math. 88 (2007), 153-163. 1

[2] D. Constales, R. De Almeida and R. S. Krausshar, On the relation between the growth and the Taylor coefficients of entire solutions to the higher dimensional Cauchy-Riemann system in $\mathbb{R}^{n+1}$, J. Math. Anal. App. 327 (2007), 763-775. 12

[3] G. P. Kapoor and A. Nautiyal, Polynomial approximation of an entire function of slow growth, J. Approx. Theory 32 (1981), 64-75. 1

[4] M. N. Seremeta, On the connection between the growth of the maximum modulus of an entire function and the moduli of the coefficients of its power series expansion, Amer. Math. Soc. Trans. 88 (1970), 291-301. 1. 